\title{
The flow field downstream of a hydraulic jump
}

\author{
By HANS G. HORNUNG, CHRISTIAN WILLERT \\ AND STEWART TURNER $\dagger$
}

Graduate Aeronautical Lab., California Institute of Technology, Pasadena, CA 91125, USA

(Received 15 April 1994 and in revised form 13 August 1994)

A control-volume analysis of a hydraulic jump is used to obtain the mean vorticity downstream of the jump as a function of the Froude number. To do this it is necessary to include the conservation of angular momentum. The mean vorticity increases from zero as the cube of Froude number minus one, and, in dimensionless form, approaches a constant at large Froude number. Digital particle imaging velocimetry was applied to travelling hydraulic jumps giving centre-plane velocity field images at a frequency of $15 \mathrm{~Hz}$ over a Froude number range of $2-6$. The mean vorticity determined from these images confirms the control-volume prediction to within the accuracy of the experiment. The flow field measurements show that a strong shear layer is formed at the toe of the wave, and extends almost horizontally downstream, separating from the free surface at the toe. Various vorticity generation mechanisms are discussed.

\section{Introduction}

At sufficiently high Froude number, the flow downstream of a hydraulic jump is so obviously rotational that it can be seen with the naked eye. The mechanism of vorticity generation is, however, still controversial. There is, of course, a source of vorticity at the solid boundary below the bottom fluid. The sign of the vorticity generated there depends on whether this boundary is stationary relative to the jump or relative to the upstream lower fluid. This vorticity stays within the boundary layer, however, and is not the concern here.

In a recent publication, Yeh (1991) discusses mechanisms of vorticity generation in a steady-flow hydraulic jump. Three contributions are identified: viscous shear at the interface between the two fluids, the baroclinic torque brought about by the static pressure gradient in the upper fluid, and the baroclinic torque brought about by the dynamic pressure gradient associated with a suitable velocity field in the upper fluid. The last of these is shown to dominate the other two and is proportional to the density ratio. It requires the vertical component of the pressure gradient in the upper fluid to be of opposite sign to that corresponding to the static gradient, so that a significant velocity field with prescribed features has to be present in the upper fluid. Since this velocity field will depend on whether the far-field upper fluid is at rest relative to the wave or relative to the upstream lower fluid, the vorticity generation would be different in these two cases.

When the density ratio is very large, such as for an air/water interface, where it is approximately 1000, such a dependence on the motion of the tenuous upper fluid seems too sensitive. One might carry the argument to the extreme case of a

$\dagger$ Permanent address: Research School of Earth Sciences, Australian National University, Canberra, Australia 
mercury vapour/liquid interface, and ask whether the vapour motion would be able to influence the vorticity downstream of a hydraulic jump in the liquid. In that case the density ratio is $O\left(10^{7}\right)$, and Yeh's theory would imply that the dimensionless vorticity would be 10000 times as large in the mercury case than in the water/air case at the same Froude number, which is not physically reasonable.

The present investigation was motivated by this difficulty. The fact that the momentum vector of the fluid entering the jump and the momentum vector of the fluid leaving it are not collinear suggested the application of the conservation of angular momentum to a control volume surrounding the jump. In this manner it was hoped that, just as in the classical derivation of the jump conditions, the omission of the conservation of mechanical energy would allow unresolved dissipative processes in the control volume to occur, and yet permit the jump conditions and the mean downstream vorticity to be determined. It also does not preclude unsteady processes within the control volume. The aim of the first part of this work is thus to consider a steady-flow hydraulic jump in a constant-density fluid on a horizontal, frictionless, solid surface, when the fluid has a density very much larger than that of the overlying fluid, so that the pressure at the free surface may be considered to be uniform, with a view to determining the mean vorticity downstream of the jump by the application of the conservation of angular momentum. This approach leaves the mechanism of vorticity generation unspecified. With these assumptions, the velocities of the far-field upper fluid and of the solid bottom relative to the jump are irrelevant. This part of the work was previously presented at a conference by Hornung (1992).

In a second part, the aim is to test the predictions of this theoretical analysis by experiment. The digital particle-imaging velocimetry set-up developed by Gharib and his co-workers was made available for this purpose. The hydraulic jump was generated in the water channel operated by Dr F. Raichlen at the Keck Laboratory at Caltech, which is ideal for the purpose.

The paper concludes with a discussion of possible mechanisms for the generation of vorticity in the hydraulic jump.

\section{Control-volume analysis}

\subsection{The classical jump conditions}

The classical equations connecting the conditions upstream and downstream of a hydraulic jump are derived from a consideration of the conservation of mass and momentum in a control volume reaching to regions upstream and downstream where the flow is considered to be uniform. This derivation is repeated here as a form of introduction of the variables of the problem. Figure 1 shows the control volume of the classical situation, with uniform velocity profiles upstream and downstream of the jump.

In terms of the quantities defined by Figure 1, the conservation of mass across the jump is ensured if

$$
h_{1} U_{1}=h_{2} U_{2} \text {, }
$$

or

$$
\frac{h_{2}}{h_{1}}=\frac{U_{1}}{U_{2}} .
$$

Similarly, the conservation of momentum requires (in the absence of friction on the 


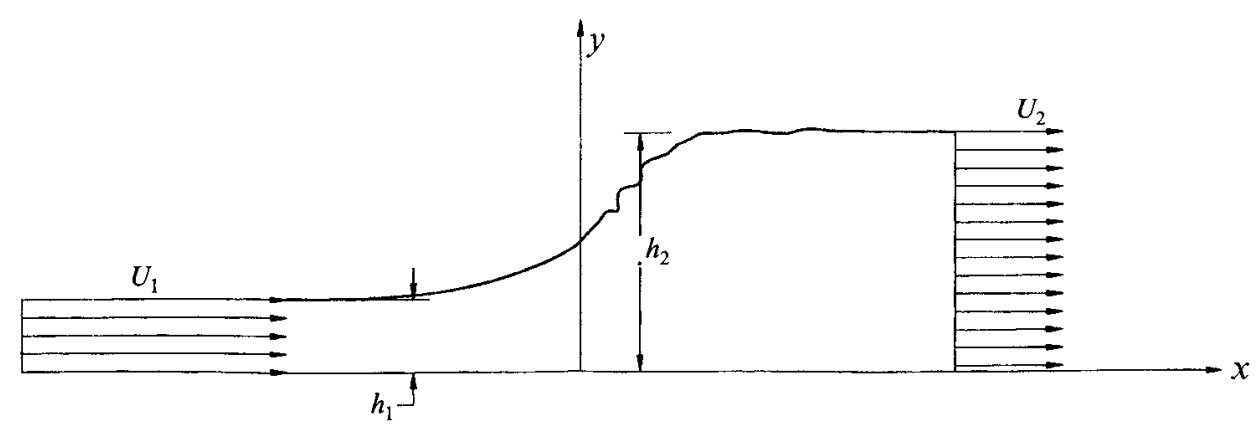

FIGURE 1. Schematic sketch of hydraulic jump with control volume.

bottom) that

$$
\frac{\rho g h_{1}^{2}}{2}+\rho U_{1}^{2} h_{1}=\frac{\rho g h_{2}^{2}}{2}+\rho U_{2}^{2} h_{2}
$$

or, manipulating this by using (1),

$$
\frac{U_{1}^{2}}{g h_{1}}=\frac{1}{2} \frac{h_{2}^{2}}{h_{1}^{2}}\left(1+\frac{h_{1}}{h_{2}}\right)
$$

Now introduce the definitions of the Froude number $F$ :

$$
F=\frac{U_{1}^{2}}{g h_{1}}
$$

and the height ratio $H$ :

$$
H=\frac{h_{2}}{h_{1}},
$$

in order to rewrite equation (2.2) in the form

$$
F=\frac{H^{2}}{2}\left(1+\frac{1}{H}\right)
$$

with the limits $H \rightarrow 1$ as $F \rightarrow 1$, and $H \rightarrow(2 F)^{1 / 2}$ as $F \rightarrow \infty$.

\subsection{Jump conditions with downstream vorticity}

We now anticipate that the velocity profile downstream of the jump will be rotational and give it not only a mean velocity $U_{2}$, but in addition a mean vorticity $\omega$ (figure 2). Thus the velocity distribution on the downstream side of the jump is now written as

$$
U=U_{2}+\omega\left(\frac{h_{2}}{2}-y\right)
$$

where $y$ is the distance from the horizontal solid bottom, measured vertically upward. Note that this does not mean that the control-volume analysis is only valid if the downstream vorticity is uniform. It merely means that the control-volume analysis can only determine the average value of the vorticity, whatever its distribution may be.

This change does not affect the mass balance, but the momentum balance has to be modified. It now requires that

$$
\frac{g h_{1}}{U_{1}^{2}}+2=\frac{h_{2}^{2}}{h_{1}^{2}} \frac{g h_{1}}{U_{1}^{2}}+2 \frac{h_{1}}{h_{2}}+I
$$




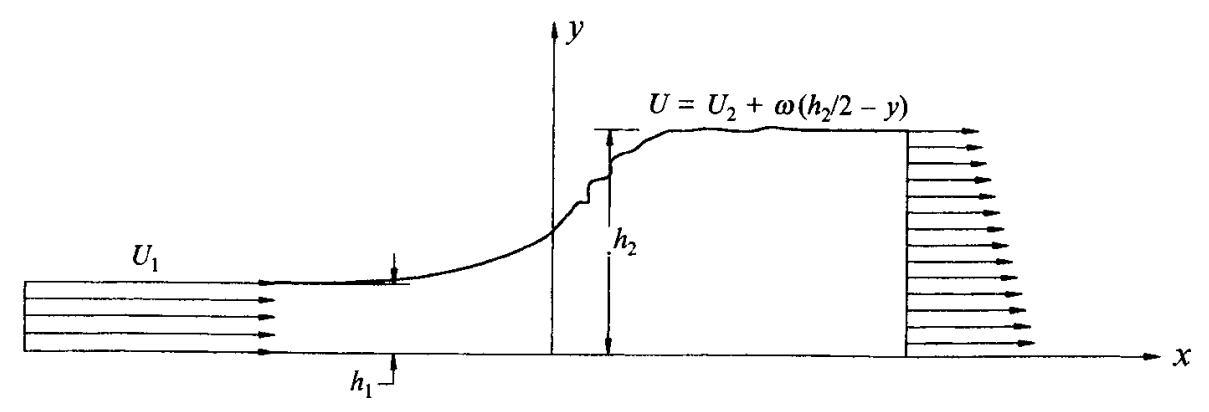

FIGURE 2. Schematic sketch of hydraulic jump with control volume for the case with finite mean vorticity downstream

where

$$
I=2 \frac{h_{1}}{h_{2}} \int_{0}^{1}\left[\frac{2 \omega}{U_{2}}\left(\frac{h_{2}}{2}-y\right)+\frac{\omega^{2}}{U_{2}^{2}}\left(\frac{h_{2}}{2}-y\right)^{2}\right] \mathrm{d}\left(\frac{y}{h_{2}}\right)=\frac{1}{6} \frac{\Omega^{2}}{H},
$$

where

$$
\Omega=\frac{\omega h_{2}}{U_{2}}
$$

Substituting this back into the momentum balance and writing the new result in the dimensionless variables, we obtain the new relation

$$
\frac{H^{2}}{2 F}\left(1+\frac{1}{H}\right)=1-\frac{\Omega^{2}}{12(H-1)} .
$$

The difference between the previous result, equation (2.5) and this is that a new term in $\Omega^{2}$ appears on the right. $\Omega$ is of course not known until a new condition is applied.

The appropriate new condition is the conservation of angular momentum, which demands that the torque applied to the control volume by external forces be equal to the rate of change of the angular momentum of the fluid contained in it. The procedure is considerably more complex than in the conservation of linear momentum, because the vertical forces on the control volume, which are balanced in the linear momentum equation, produce a torque.

\subsubsection{The torque arising from vertical forces}

If the vertical components of the inertial forces were zero, the bottom pressure would exactly balance the weight of the fluid, so that no net torque would be exerted by the vertical force components. In the left half of the control volume there is a mean concave-up streamline curvature. To provide this curvature a transverse pressure gradient is required. Since the pressure at the free surface is independent of streamwise distance (assuming the density of the overlying fluid to be negligible) the pressure on the bottom must exceed the static pressure corresponding to the height of liquid above it. The opposite situation occurs in the downstream half of the control volume, where the mean streamline curvature is convex up, so that the bottom pressure is lower than it would be without this inertial force.

The additional bottom pressure distribution brought about by the vertical acceleration of the fluid is thus antisymmetric in $x$, and will exert a clockwise torque on the fluid. This also has the required feature that it disappears at $F \rightarrow 1$, since the mean 
streamline curvature disappears, and increases as $F$ increases. Unfortunately, it is not possible to obtain it without some further assumptions. Let this torque be $t$ per unit lateral distance and introduce the dimensionless torque

$$
T=\frac{t}{\rho U_{1}^{2} h_{1}^{2}} .
$$

To determine $T$, consider the differential form of the continuity and vertical momentum equations:

$$
\begin{gathered}
\frac{\partial u}{\partial x}+\frac{\partial v}{\partial y}=0 \\
u \frac{\partial v}{\partial x}+v \frac{\partial v}{\partial y}=-\frac{1}{\rho} \frac{\partial p}{\partial y}-g,
\end{gathered}
$$

where the symbols have their usual meaning. By using the continuity equation to replace $\partial v / \partial y$ in the momentum equation, and replacing $p$ with the excess pressure $p^{\prime}$ over the static pressure according to

$$
p^{\prime}=p-\rho g(h-y),
$$

the momentum equation becomes

$$
-\frac{1}{\rho} \frac{\partial p^{\prime}}{\partial y}=u \frac{\partial v}{\partial x}-v \frac{\partial u}{\partial x}=u^{2} \frac{\partial}{\partial x}\left(\frac{v}{u}\right) .
$$

At the free surface,

Assume that

$$
v(x, h)=u(x, h) \frac{\mathrm{d} h}{\mathrm{~d} x} .
$$

$$
u h(x)=U_{1} h_{1}
$$

i.e. independent of $y$, and

$$
v=v(x, h) \frac{y}{h}=U_{1} h_{1} \frac{\mathrm{d} h}{\mathrm{~d} x} \frac{y}{h^{2}},
$$

i.e. a linear profile satisfying the bottom condition $v(x, 0)=0$. Substituting these in equation (2.10), we obtain

$$
-\frac{1}{\rho} \frac{\partial p^{\prime}}{\partial y}=\frac{U_{1}^{2} h_{1}^{2} y}{h^{2}} \frac{\mathrm{d}}{\mathrm{d} x}\left(\frac{1}{h} \frac{\mathrm{d} h}{\mathrm{~d} x}\right) .
$$

This may be integrated over $y$ from 0 to $h$ to give the excess bottom pressure:

$$
2 p_{b}^{\prime}=-\rho U_{1}^{2} h_{1}^{2} \frac{\mathrm{d}}{\mathrm{d} x}\left(\frac{1}{h} \frac{\mathrm{d} h}{\mathrm{~d} x}\right) .
$$

Consequently the clockwise torque per unit transverse distance exerted by the excess bottom pressure on the fluid is

$$
T=\frac{t}{\rho U_{1}^{2} h_{1}^{2}}=-\frac{1}{2} \int_{-\infty}^{\infty} x \frac{\mathrm{d}}{\mathrm{d} x}\left(\frac{1}{h} \frac{\mathrm{d} h}{\mathrm{~d} x}\right) \mathrm{d} x .
$$

Figure 3 plots the excess pressure as a function of dimensionless distance for the case of a hyperbolic-tangent wave shape.

However, it is in fact not necessary to assume a particular wave shape, because 


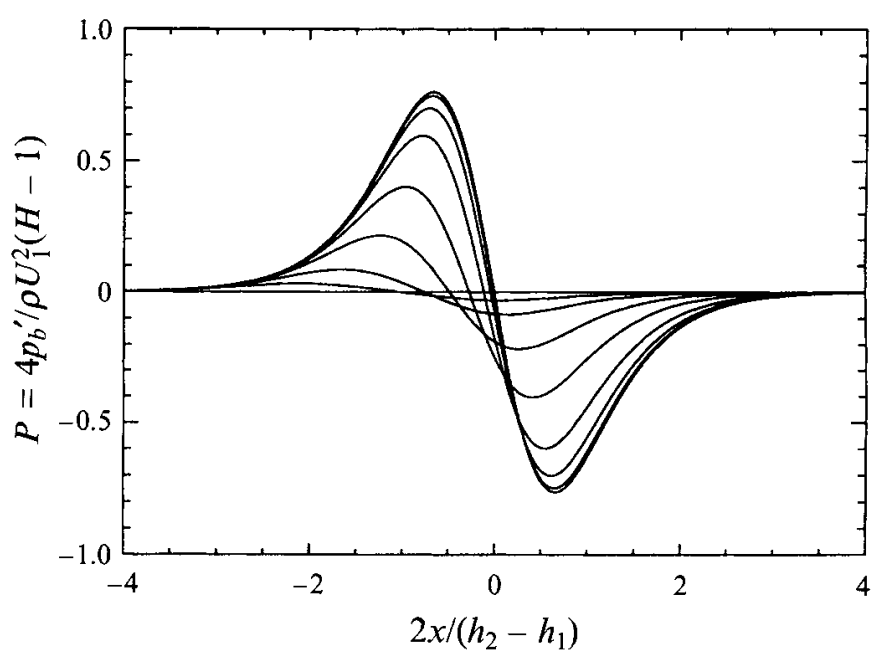

FiguRE 3. The behaviour of the excess pressure (scaled by $(H-1) / 2$ ) for a hyperbolic-tangent wave shape and values of $H-1=0.01,0.03,0.1,0.3,1,3,10$, and 30 . In this representation the largest amplitude corresponds to the smallest value of $H$.

(11) may be integrated by parts to give

$$
T=-\frac{1}{2}\left[\frac{x}{h} \frac{\mathrm{d} h}{\mathrm{~d} x}-\ln h\right]_{-\infty}^{\infty} .
$$

For all wave shapes of interest to us,

$$
\frac{x}{h} \frac{\mathrm{d} h}{\mathrm{~d} x} \rightarrow 0 \quad \text { as } \quad x \rightarrow \pm \infty
$$

and

$$
T=\frac{1}{2} \ln \frac{h_{2}}{h_{1}}=\frac{1}{2} \ln H .
$$

To the approximation made here, the torque from the excess bottom pressure is thus independent of the detail of the wave shape. This may be expected to be correct for sufficiently small values of $H$, but care needs to be exercised at larger $H$, because the assumptions made earlier about the forms of $u(x, y)$ and $v(x, y)$ break down as $H \rightarrow \infty$. One cause of this failure is evident from a consideration of the situation when the spill of the wave forms the roller that is observed at large $H$ in a hydraulic jump.

It is important to check that the excess bottom pressure does not produce a net force. This may easily be done by integrating the pressure distribution over $x$. The result is

which is zero.

$$
\int_{-\infty}^{\infty}-\frac{2 p_{b}^{\prime}}{\rho U_{1}^{2} h_{1}^{2}} \mathrm{~d} x=\left[\frac{1}{h} \frac{\mathrm{d} h}{\mathrm{~d} x}\right]_{-\infty}^{\infty},
$$

\subsubsection{Angular momentum balance}

The torque exerted by the bottom pressure distribution may now be used, together with the terms arising from horizontal forces and momenta, in an angular momentum balance on the control volume. Taking moments about a point, e.g. in the bottom 


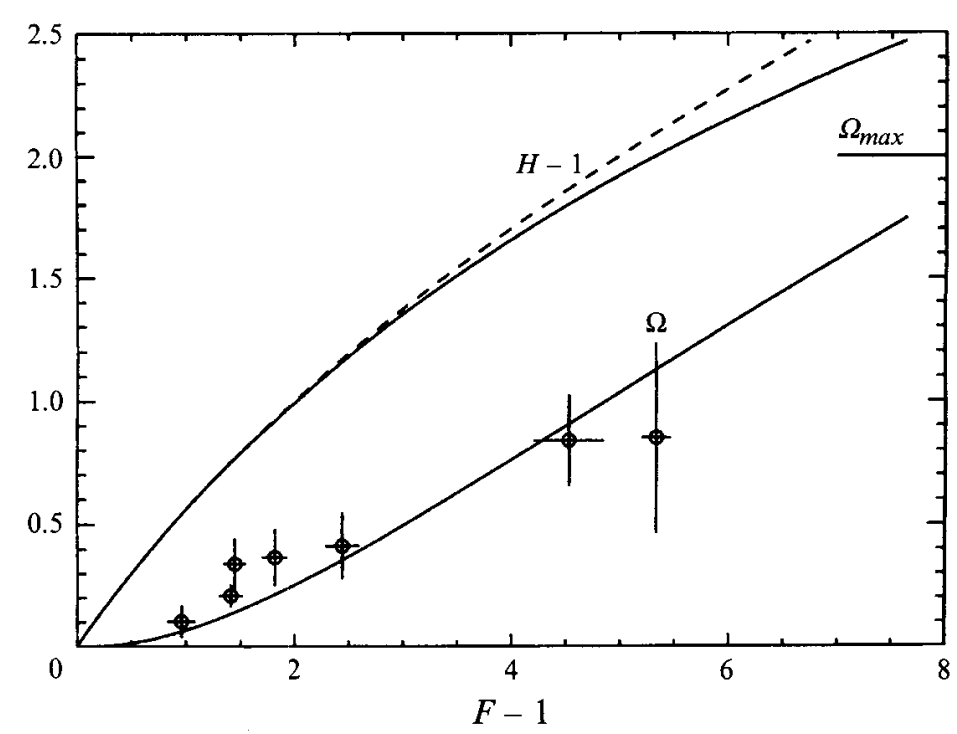

FIgURE 4. The wave height in the form $H-1$ and the downstream vorticity $\Omega$ as functions of the Froude number $(F-1)$ as given by simultaneous solution of (9) and (15). As may be seen, $H-1$ increases linearly with $F-1$ near $F=1$ in the same way as without downstream vorticity (dashed curve gives classical result, equation (2.5)). Also, $\Omega$ increases from zero as the cube of $F-1$. The experimental points will be discussed in $\S 4.1$. Note: The difference between the dashed and full lines for $H-1$ remains small as $F$ increases, since $\Omega$ is limited to 2 .

surface (the location of the point is immaterial, because of the horizontal momentum balance and the absence of net vertical force), angular momentum balance yields

$$
\frac{g h_{1}{ }^{3}}{6}+\frac{U_{1}^{2} h_{1}^{2}}{2}+h_{1}^{2} U_{1}^{2} T=\frac{g h_{2}{ }^{3}}{6}+\frac{U_{2}^{2} h_{2}^{2}}{2}+\int_{0}^{h_{2}}\left(2 U_{2}\left(U-U_{2}\right)+\left(U-U_{2}\right)^{2}\right) y \mathrm{~d} y .
$$

Substituting for $U$ from (2.6) and writing the result in dimensionless form, this becomes

$$
\frac{1-H^{3}}{F}=\Omega\left(\frac{\Omega}{4}-1\right)+6 T
$$

Substituting for $T$, and solving for $\Omega$,

$$
\Omega=2\left[1-\left(1-\frac{H^{3}-1}{F}+3 \ln H\right)^{1 / 2}\right] .
$$

$\Omega(F)$ and $H(F)$ may now be obtained by solving (9) and (15) simultaneously. The result is plotted in figure 4 .

The behaviour of $\Omega$ in the limits $F \rightarrow \infty$ and $F \rightarrow 1$ exhibits interesting features. At $F-1 \rightarrow 0$ series solutions may easily be obtained for $F-1$ and $\Omega$ in increasing powers of $\epsilon=H-1$. The results are

$$
\Omega=\frac{3}{4} \epsilon^{3}-\frac{9}{8} \epsilon^{4}+\frac{107}{80} \epsilon^{5}+O\left(\epsilon^{6}\right),
$$

and

$$
F-1=\frac{3}{2} \epsilon+\frac{1}{2} \epsilon^{2}+\frac{3}{64} \epsilon^{5}+O\left(\epsilon^{6}\right) .
$$


Thus the vorticity increases from zero as

$$
\Omega=\frac{2}{9}(F-1)^{3}+O(F-1)^{4} .
$$

This is reminiscent of the manner in which dissipative effects behave in the analogous situation of a shock wave in a compressible fluid. The square of the Mach number $M^{2}$ corresponds to the Froude number $F$ in the analogy and dissipative effects manifest themselves in the form of an entropy increase in the shock wave. This entropy change increases with the cube of $M^{2}-1$, just as the manifestation of dissipative effects in the hydraulic jump (namely vorticity) increases as the cube of $F-1$ here.

At the other extreme, the present solutions are not able to make a satisfactory prediction, because the approximations leading to the expression for the torque from the bottom pressure break down. However, it is possible to make an intelligent guess about the behaviour of the expression under the square root of equation (2.15). The trend of this term is to decrease towards zero at $F \approx 9.5$. It must not change sign, however, if the solution is to remain real. We expect, therefore, that the physical case corresponds to the term approaching zero smoothly as $F \rightarrow \infty$. If this is correct, $\Omega$ will approach the value 2 asymptotically. This is also the maximum value that can be reached, because it corresponds to $U\left(h_{2}\right)=0$. Larger values of $\Omega$ imply negative $U\left(h_{2}\right)$, which does not make sense, because it corresponds to the downstream fluid overtaking the wave.

It should be noted that the effect of finite $\Omega$ on the height ratio and propagation speed remains small at large $F$. This may also be seen from the fact that the last term in equation (2.9) remains small if $\Omega \rightarrow 0$ as $F \rightarrow \infty$.

The value of $\Omega$ at $F \rightarrow \infty$ may be approximately assessed from the experimental evidence that a surfboard or other floating object is carried along almost at the same speed with a large broken wave even if it is just downstream of the wave. This indicates that the flow speed at the top and just downstream of a large broken wave is the same as that of the wave itself. This corresponds to $\Omega=2$, and lends additional support to our guess.

\section{Experiment}

\subsection{The water channel}

The water channel at the Keck Laboratory at Caltech was used for the experiments. We studied the travelling hydraulic jump, or bore, produced by suddenly opening a gate between two stationary bodies of water at different levels. The channel is shown schematically in figure 5 in the two configurations just before and shortly after the gate is opened.

During the experiment, the height ratios and Froude number were measured and compared with the classical theory. Equation (2.5) and the predicted height ratio upstream and downstream of the jump (which is a function the height ratio before opening the gate) were confirmed to within the experimental accuracy which was estimated as $\pm 2 \%$ in the height ratios and $\pm 6 \%$ in the Froude number in the range from $2<F<6$.

\subsection{The DPIV set-up}

The Digital Particle Imaging Velocimetry (DPIV) set-up is sketched schematically in figure 6. In the arrangement used the particles were silver-coated glass spheres of $10 \mu \mathrm{m}$ diameter with a density of $1100 \mathrm{~kg} \mathrm{~m}^{-3}$, giving a vertical sedimentation speed 

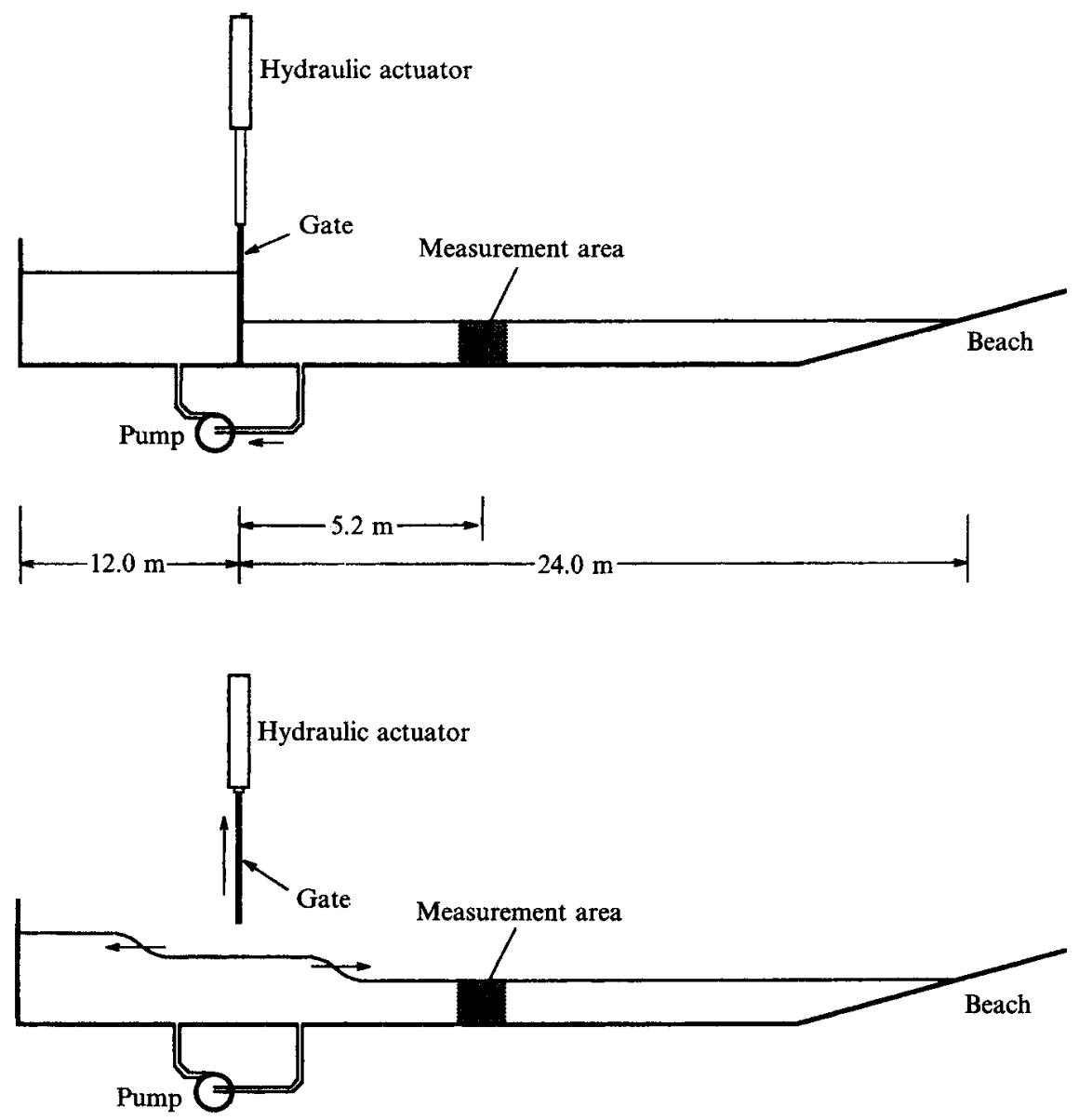

FIGURE 5. Schematic sketch of the water channel in the Keck Laboratory

under gravity of approximately $0.1 \mathrm{~mm} \mathrm{~min}^{-1}$. The flow speeds in the experiments are typically $0.3 \mathrm{~m} \mathrm{~s}^{-1}$.

The particles are approximately uniformly dispersed before the flow starts. They are illuminated with an continuous argon-ion laser in a sheet along the vertical centre-plane of the channel as shown. The sheet is approximately $1 \mathrm{~mm}$ thick. The sheet is pulsed with a shutter to produce pulse pairs of $1.5 \mathrm{~ms}$ duration separated by $5 \mathrm{~ms}$. These are used to acquire velocity fields at $15 \mathrm{~Hz}$. The particle images are recorded with a Texas Instruments MC-1134 CCD camera using a $37.5 \mathrm{~mm} f 1.1$ lens as analog video records on a Sony laser video disk. A period of $10 \mathrm{~s}$ is recorded each run, giving 150 image pairs per run. The data are then digitized with a PC frame grabber and DPIV-processed on a SPARC-station. Typical particle displacements measured were in the range $1-5 \mathrm{~mm}$, corresponding to 5-25 pixels. The field of view was $100 \mathrm{~mm}$ wide and $130 \mathrm{~mm}$ high. The tank configuration used, with the gate positioned one third of the length from the left-hand (deep) end, had a useful geometrical consequence. The upstream depth varied by less than $10 \%$ over the whole range of depth ratios investigated, so that the same measurement area could be used in all the experiments on strong jumps. The accuracy of velocity and vorticity determination is estimated to be typically $\pm 2 \%$ and $\pm 10 \%$ respectively. 


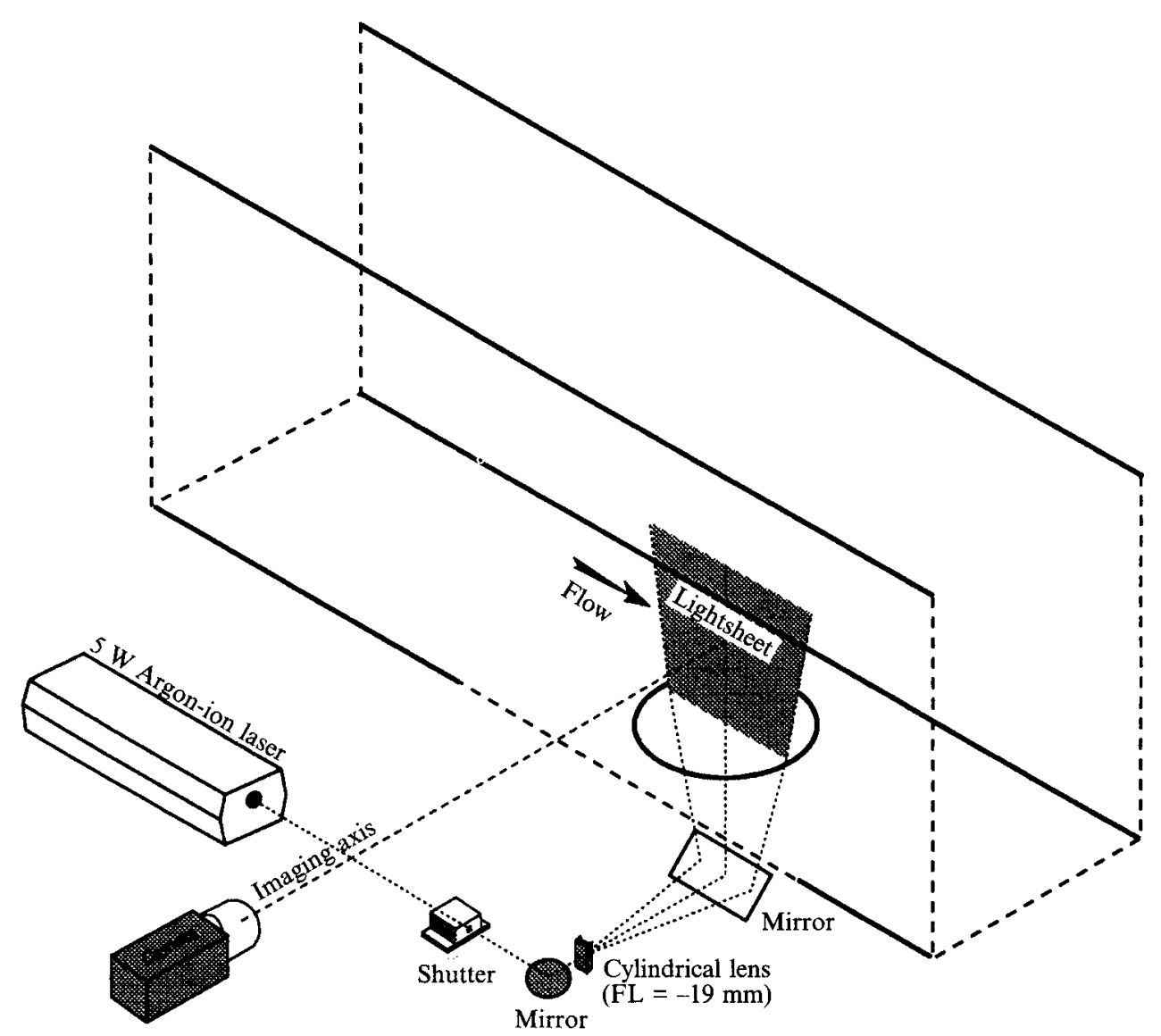

FIGURE 6. Sketch of DPIV optics arrangement in relation to the water channel

The experiments also confirmed the standard predictions about the nature of the jumps for various height ratios $H$. For $H<1.35$, corresponding to $F<1.6$, the jumps were wavy; $1.35<H<1.5$ and $1.6<F<1.9$ was a transition regime, with a large wave and a small breaking region, and above $H=1.5$ or $F=1.9$ the jumps were fully turbulent. It is this last regime to which the theory should be applicable, and in which all the analysed runs lay. The difference between the height ratio predicted by the theory with downstream mean vorticity and without it, see figure 4 , is too small to be resolved by the experiment.

The method of obtaining the velocity field from the particle images is described in detail by Willert \& Gharib (1991).

\subsection{Sample experimental results}

In all, 28 runs were recorded in the manner described. A number of these suffered from shutter defects or faulty adjustment, and others were wavy, rather than fully turbulent, jumps as described above, so that only ten of these produced data, in the Froude number range of interest here, that could be analysed in detail to obtain velocity fields. These cover the range of Froude number from 2 to 6 . An example of the instantaneous velocity field after a jump with $F=2.4$ is shown in figure 7 . The velocity obtained by associating particle image pairs is averaged over a sampling 


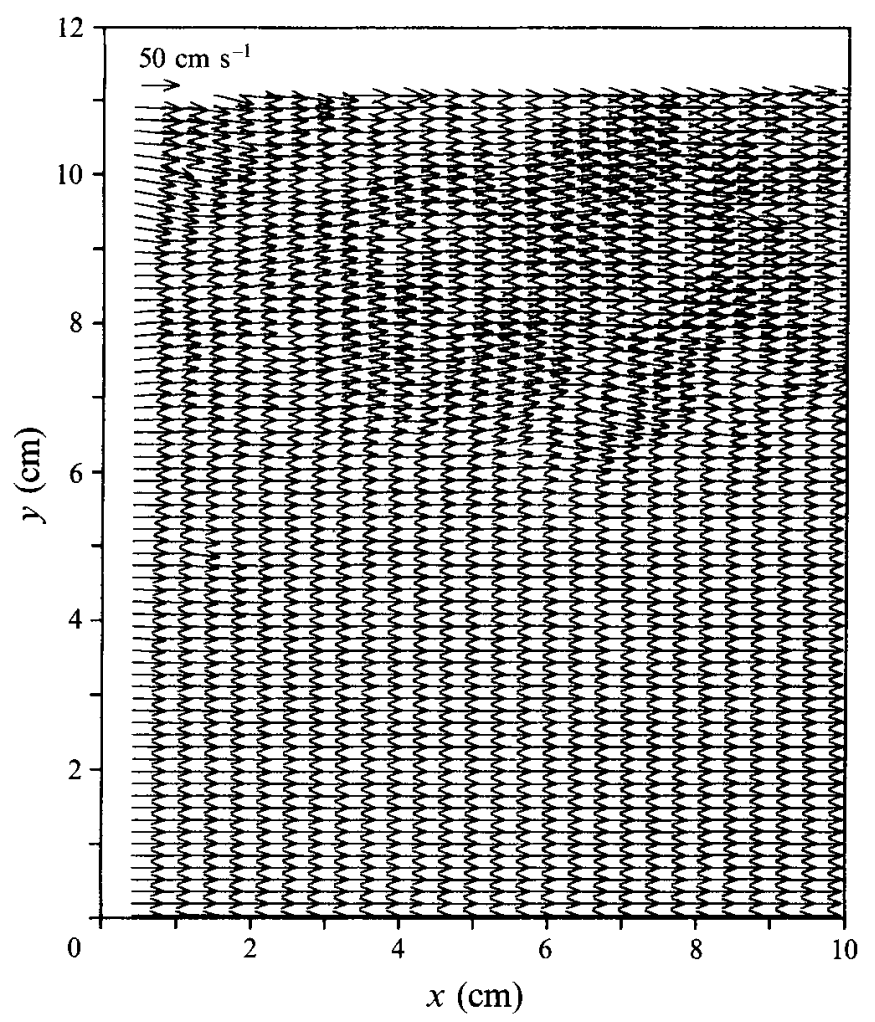

FIGURE 7. Example of the velocity field measurements

area of $1.6 \times 1.6 \mathrm{~mm}$, and plotted in the figure in the form of a rectangular array of arrows, whose length is proportional to the velocity magnitude (see scale at top left), and pointing in the local flow direction. This is the velocity measured relative to the laboratory frame, in which the jump travels from left to right.

In order to relate the velocity to the jump-fixed coordinates used in the controlvolume analysis with the flow coming from the left, this picture therefore would have to be laterally inverted and the jump speed would have to be subtracted from the velocity vectors. In order to bring out the turbulent structure of this flow, figure 8 shows the velocity vectors that would be seen by an observer travelling with the average velocity of this frame. It now becomes clear that the upper half of the flow field shows strong turbulent structures which have an average velocity toward the jump, which has disappeared out of the field of view to the right at the time of the measurement. The mean vorticity is therefore of the same sign as that predicted by the control-volume analysis.

A very interesting feature of the flow field is that the lower part of the frame exhibits approximately uniform flow, while only the upper part exhibits a mean shear and turbulent structure.

When the Froude number is large, the region near the free surface contains entrained air bubbles which strongly reflect the laser light, thus making velocity evaluation impossible in their vicinity. 


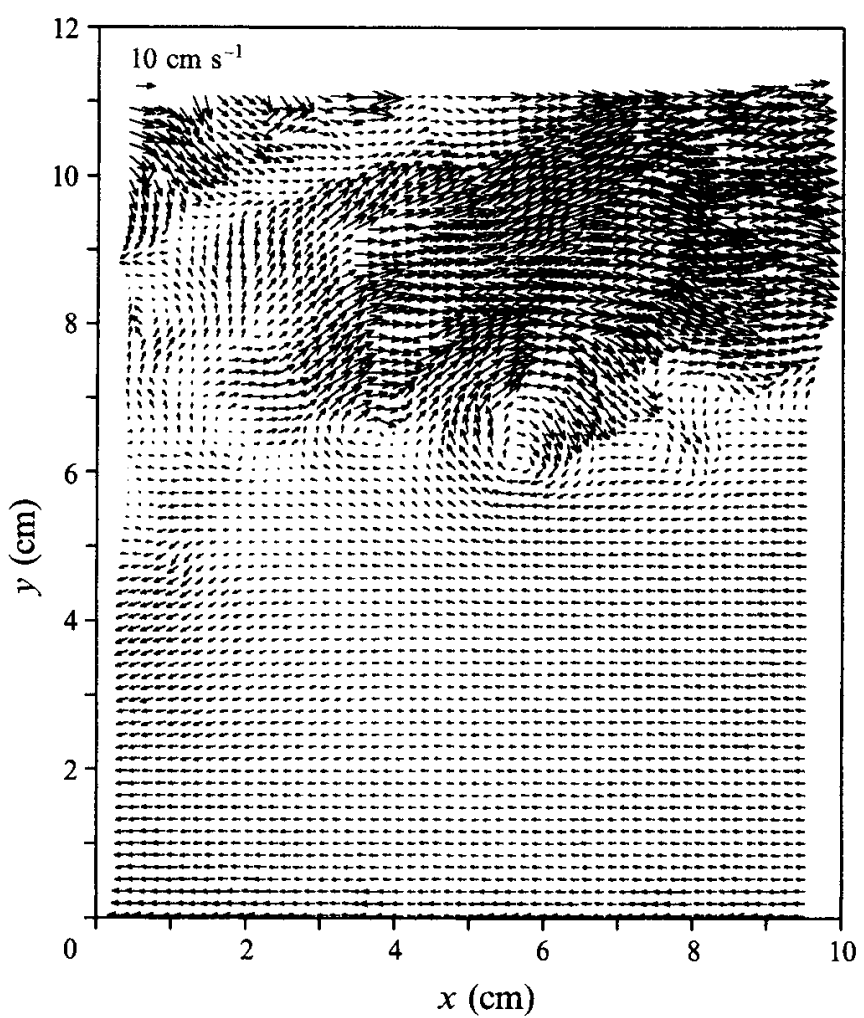

FIGURE 8 . Velocity field as seen by an observer moving with the average velocity

\section{Data analysis and discussion}

\subsection{Mean vorticity}

The experimental study provides a great deal more information than is required to test the prediction of the control-volume analysis. However, we discuss this test first.

In fact, it is quite difficult to obtain an accurate determination of the mean vorticity from the data. The average of the vorticity over the vertical distance is the difference between the velocities at the top and at the bottom divided by the height. However, the velocity measurement at the top suffers from the inaccuracy resulting from the reflections from entrained air bubbles, especially at larger $F$, and, at the bottom, the floor boundary layer, which is ignored in the control volume analysis, introduces a further uncertainty.

One method of determining an average vorticity from a particular frame such as that in figure 7 is to average the velocity first along the horizontal lines of the grid, to give a velocity profile $u(y)$, and then to form the average vorticity from

$$
\omega_{2}=\frac{u\left(h_{2}\right)-u(0)}{h_{2}} \text {. }
$$

Figure 9 shows the result of doing this in the case of Run 15 for five frames at different times after the passage of the wave.

In order to provide a value of $\omega_{2}$ that is suitable for comparison with the controlvolume analysis, $u(0)$ is measured at the edge of the floor boundary layer and $u\left(h_{2}\right)$ is taken to be the value at the top of the measurable region. As may be seen by 


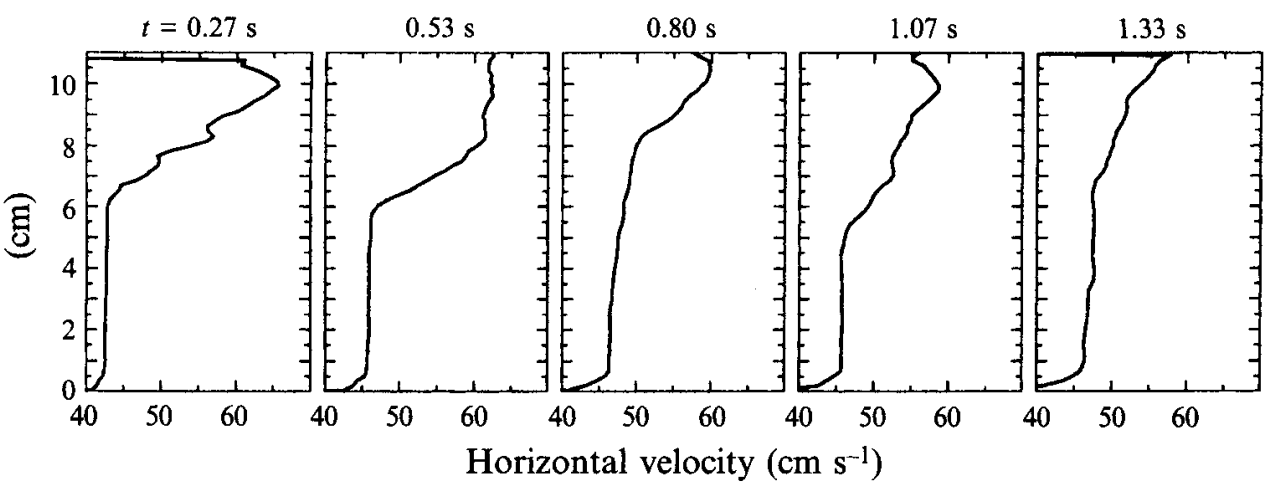

FIGURE 9. Horizontally averaged velocity profiles at different times after passage of the wave. Run 15.

inspection of figure 9 , the resulting value will fluctuate quite significantly over the time covered. It is necessary to limit the time over which the vorticity is averaged. This is because the interface between the water that was originally on the high side of the gate and the water that was originally on the low side of the gate is at a finite distance behind the jump. Only the fluid that is ahead of this interface has been processed by the jump. By averaging the value of $\omega_{2}$ over the time up to the arrival of the interface, a suitable average and variance can be determined.

Alternatively, an average vorticity can be obtained by performing a line integral around the outside of the field of view and dividing by the area. Although this seems to have some appeal, because the area may be chosen to lie within the uncertain top and bottom edges of the field of view, it is also dangerous, because the method might exclude local maxima of the vorticity if the field chosen is too small.

Both methods were used, and the former seemed to provide smaller variance, though the results do not display any other significant differences. In order to provide a comparison with the control-volume prediction, the experimental results together with error bars that indicate the scatter, are superimposed on figure 4. As may be seen, the experimental results give approximate agreement with the control-volume argument, although the data could equally well be fitted by a straight line as by the predicted curve.

\subsection{Flow-field structure and evolution}

The power of the DPIV capability is brought out by studying a sequence of consecutive frames of the velocity field measurements in one run starting from the time of arrival of the jump. Frame pairs of DPIV images were taken at $15 \mathrm{~Hz}$. The frames of a pair are separated by $5 \mathrm{~ms}$. Thus, the interval between the acquisition of velocity fields is $66 \mathrm{~ms}$. The method therefore provides a movie of the velocity field. Clearly, the data thus gathered are far too voluminous to be presented here. We select a run with $H=1.69$ and present the first six frames of the sequence.

Figure 10 shows the velocity fields as they would be seen by an observer moving with the approximate flow velocity in the lower part of the channel. This subtraction of the lower fluid velocity brings out the structures of the turbulent flow near the surface, and also shows the entrainment effect of these structures. Note how, in some cases, individual structures may be recognized as being the same in successive frames.

Understanding of the detail of the flow is further enhanced by studying the vorticity 

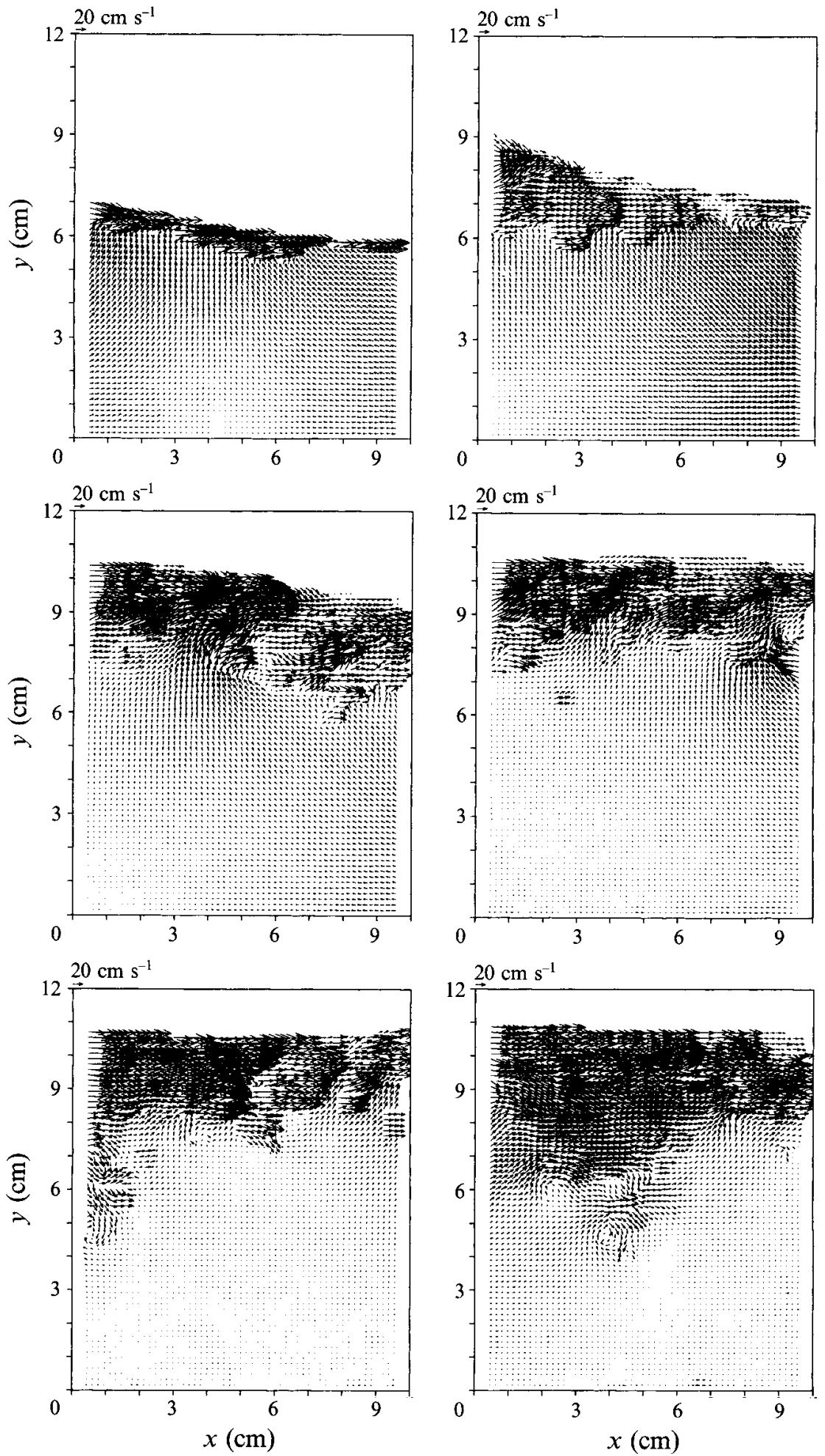

Figure 10. Evolution of velocity field, $H=1.69$. The images are separated in time by $66 \mathrm{~ms}$. Velocities of $15,39,46,50,50$ and $50 \mathrm{~cm} \mathrm{~s}^{-1}$ have been subtracted respectively from the six consecutive images. 
field. Figure 11 presents vorticity contour plots of the same six frames. Note how a strong shear layer issues from the toe of the jump (see second frame). This shear layer is unstable practically from its starting point and shows distinct vortical structures. It is clear from these pictures (see especially frame two), that the vorticity is generated at the toe of the wave. The plots are presented at the same scale as the velocity fields, so that they may be overlayed to illustrate the entrainment effect of the shear layer. This shear-layer character of the hydraulic jump has been observed previously, see Hoyt \& Sellin (1989), McCorquodale \& Khalifa (1983), and Long et al. (1991), though no detailed velocity field measurements have been made in these studies.

Above the main shear layer (see particularly the second frame) a second, weaker shear layer of opposite sign is formed. One has to be very careful about the vorticity determination at the free surface, since the entrainment of bubbles and the edge of the measurement domain cause errors there. However, the opposite-sign vorticity does appear to extend deeper into the flow than the region where bubbles are seen in photographs of the flow. The magnitude of the opposite-sign vorticity will certainly be overestimated, because the velocity at the edge of the domain is set equal to zero. In all cases the free surface is only $2-4 \mathrm{~mm}$ away from the highest measuring point, so that all but a thin layer near the surface has been resolved.

At later times the vortical structures slowly become dissipated, so that the vorticity peaks decrease and a trend to a more uniform value occurs. However, as may be seen in figure 9 , the vorticity diffuses only very slowly into the lower fluid.

The vorticity plots show that the vorticity generated by the bottom boundary layer does not influence the flow of interest at all. From the point of view of testing the control-volume analysis, the travelling jump (relative to the laboratory frame) is, in fact, a more suitable configuration than a jump that is steady in the laboratory frame, since it is difficult to produce the latter without having a relatively thick turbulent boundary layer on the bottom wall.

\section{Mechanisms of vorticity generation}

None of the discussion so far has attempted to explain the mechanism by which the vorticity is generated. Apart from the mechanism proposed by Yeh (1991), another attempt to explain vorticity generation, at a constant-pressure fluid interface without surface tension, is that proposed by Batchelor (1967) and discussed in more detail by Longuet-Higgins(1992), by which vorticity is generated at a stress-free, curved interface. This vorticity is proportional to the curvature of the interface. In the case of the hydraulic jump, interface curvatures of both signs are present. The net vorticity generated would thus be zero if the interface shape is antisymmetrical around an inflexion point. According to the control-volume analysis, the downstream vorticity is, however, substantial. The conclusion to be drawn from this is that either the wave shape is not antisymmetric so that more vorticity of one sign is generated than of the other, or a different generation mechanism exists.

We pursued possible alternative mechanisms of vorticity generation in discussions with colleagues at Caltech. One such mechanism, proposed by Dale Pullin, emerged. It may be explained as follows.

Consider a free (constant-pressure) surface with a hydraulic jump, as in $\S 1$. On the forward-facing slope of the jump small waves travelling down the slope occur if the height ratio is not too small. These small wavelets also break and entrain air. Consider such a wavelet at three stages in the steepening and breaking process, labelled 1,2 and 3, see figure 12 . 

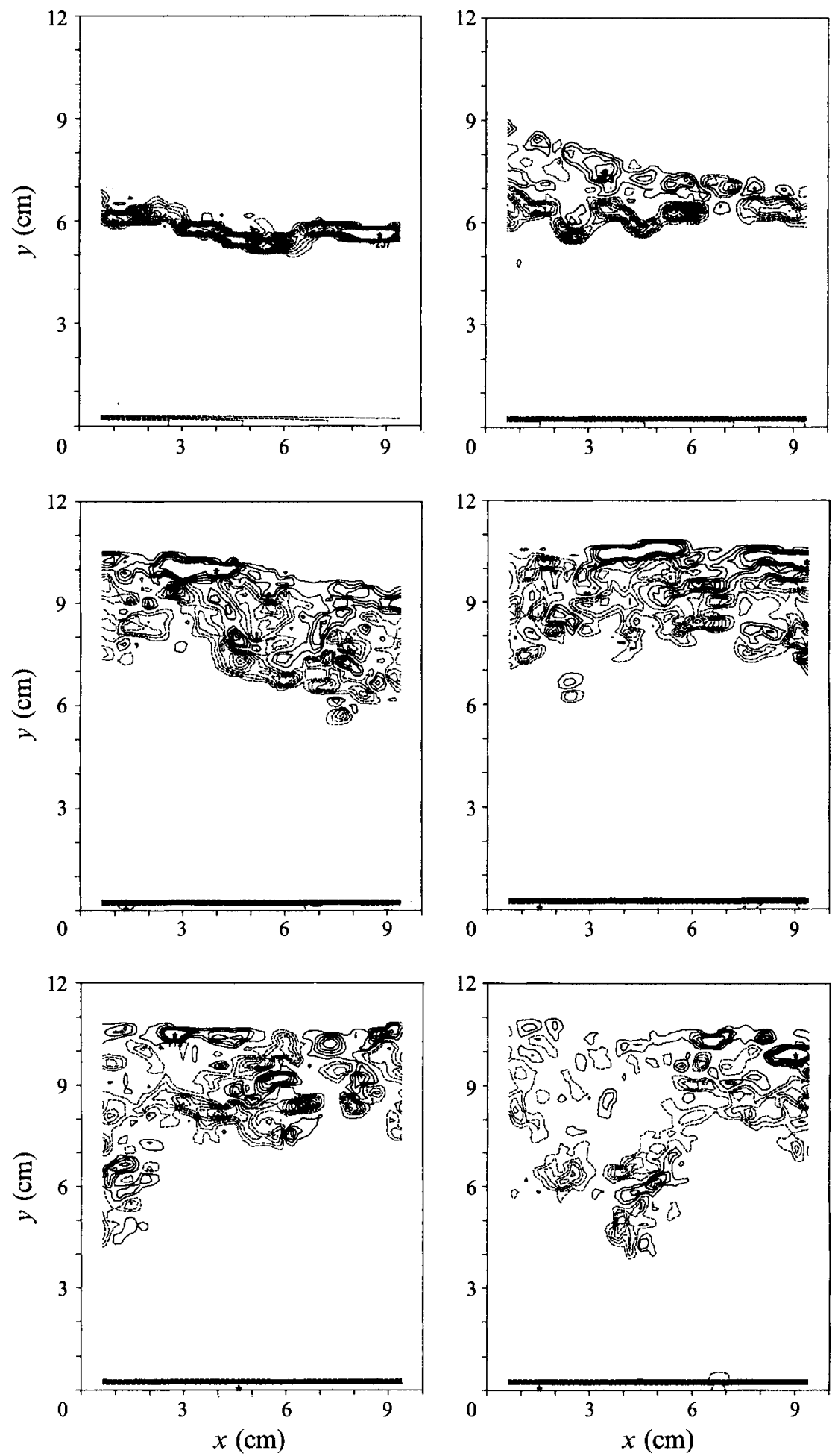

Figure 11. Evolution of the vorticity field corresponding to the velocity field of figure 10 . 


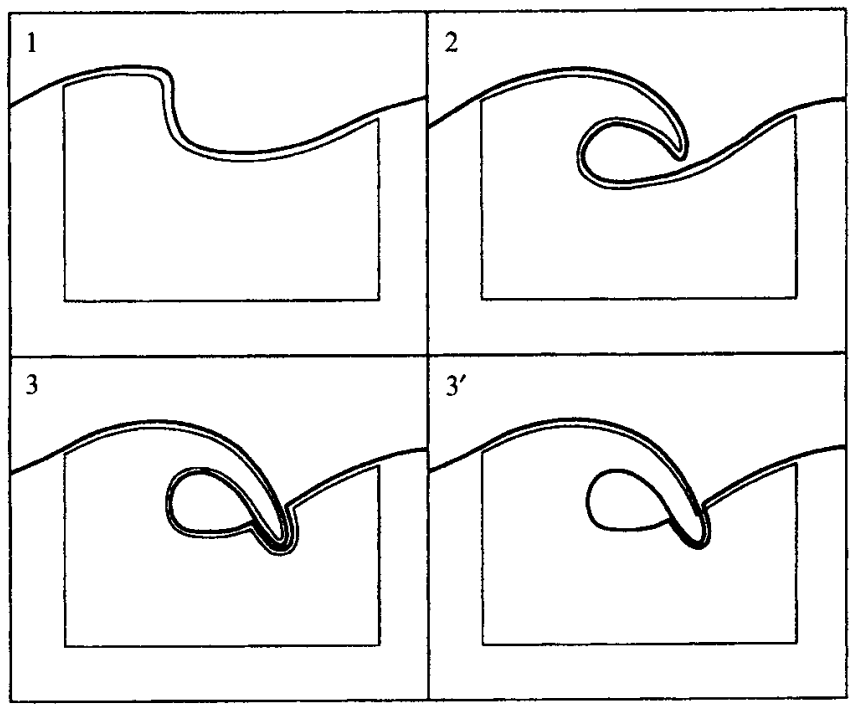

FIGURE 12. Sketch to explain vorticity generation mechanism by surface reconnection

Between 2 and 3, reconnection of the liquid occurs. We can now consider a closed material line shown in stage 1 . One segment of this line consists of the particles in the free surface, but is shown as slightly below it for clarity. Let the circulation around this line be zero. In the absence of external rotational forces, the circulation will remain zero. The material line is then deformed as shown in stages 2 and 3. In stage 3 , the line folds onto itself in such a way that two portions of it are congruent. Again, the figure shows them a little apart for clarity. The circulation around the whole loop is still zero.

We may now consider this whole loop as two separate loops, however, by connecting one of them as shown in stage $3^{\prime}$ instead. Since the circulation of the whole loop in 3 is zero, the circulation around the reconnected loop in $3^{\prime}$ is, in general, not zero. This is because the sum of the two circulations is zero (unless the velocity across the congruent interfaces is infinite at the reconnection point of the loop). The entrained bubble of upper fluid therefore has non-zero circulation around it, which can be diffused into the rest of the lower fluid while the bubble is within it. This entrainment of vorticity is thus connected with the question of where one draws the boundary of the lower fluid.

As was pointed out in the discussion of the flow field evolution, the evidence suggests that the vorticity enters at the toe of the wave, since a strong shear layer appears at that point. A discontinuity in velocity appears to be maintained there. In the frame of reference of the wave, the shallow upstream flow passes underneath the wave generating a flow field that is similar to a separation. The separation streamline is the almost horizontal shear layer.

In all the mechanisms of vorticity generation considered here, except the 'entrainment', the flow is assumed to be steady. If the free surface has a tangential component of acceleration, then a baroclinic torque term can exist, so that unsteady surface movement can create vorticity in each of the two fluids. Since unsteady surface motion is clearly in evidence in the experiment, it is a likely source of vorticity. A detailed mathematical treatment of this and other mechanisms has been presented by Wu (1993), see also Gharib et al. (1992) and Rood (1994). It is interesting to 
note that techniques such as the one used here for the velocity field measurement can, in principle, be used to test vorticity creation mechanisms, though the temporal resolution of the data $(15 \mathrm{~Hz})$ is insufficient in our case. With the separation of the shear layer at the toe, any vorticity generated on the wave face will make its way to the toe and into the shear layer by convection.

\section{Conclusions}

A control-volume analysis including the application of the conservation of angular momentum was applied to a hydraulic jump. This permitted the mean vorticity downstream of the jump to be determined as a function of Froude number. In dimensionless form (with downstream height and mean velocity) the vorticity ranges from 0 to 2 as Froude number goes from 1 to $\infty$. The height ratio is only slightly altered by the mean vorticity.

Experiments were conducted on a travelling hydraulic jump using digital particle imaging velocimetry giving successive velocity fields along the longitudinal centreplane of the jump at $66 \mathrm{~ms}$ intervals. These permitted the flow field structure and evolution to be studied in detail, as well as providing a good measurement of the mean vorticity. The latter confirmed the prediction of the control-volume analysis within the accuracy of the measurements over a Froude number range of 2-6.

The flow field measurements showed that a strong shear layer is formed at the toe of the wave and extends almost horizontally downstream, separating from the free surface at the toe. The shallow upstream flow passes underneath this shear layer and the turbulent flow of the fluid in the wave. Possible vorticity generating mechanisms were discussed, including unsteady and entrainment effects.

We wish to acknowledge the financial support of one of us (S.T.) by the Fairchild Foundation during the period of the experiments. We are also very grateful to Dr F. Raichlen for providing the use of the water channel. Dr Willert and the DPIV system were supported by an Office of Naval Research URI Grant (ONR-N00014-92-J-1610).

\section{REFERENCES}

Batchelor, G. K. 1967 An Introduction to Fluid Dynamics, p. 366. Cambridge University Press.

Gharib, M., Weigand, A., Willert, C. \& Liepmann, D. 1992 Experimental studies of vortex reconnection to a free surface. 19th Symposium on Naval Hydrodynamics. National Academy Press.

HoRnung, H. 1992 The mean vorticity downstream of a hydraulic jump. 11th Australasian Fluid Mechanics Conference, Hobart, pp. 929-932.

Hoyt, J.W. \& Sellin, R.H.J. 1989 Hydraulic jump as 'mixing layer'. Hydr. Engng, ASCE 115, $1607-1614$.

Long, D., Rajaratnam, N., Steffler, P. M. \& Smy, P. R. 1991 Structure of flow in hydraulic jumps. J. Hydr. Res. 29, 207-218.

LONGUET-Higgins, M.S. 1992 Capillary rollers and bores. J. Fluid Mech. 240, 659-679.

McCorquodale, J. A. \& Khalifa, A. 1983 Internal flow in hydraulic jumps. J. Hydr. Engng, ASCE 109, 684-701.

ROOD, E. P. 1994 Interpreting vortex interactions with a free surface. Trans. ASME I: J. Fluids Engng (to appear).

Willert, C. E. \& Gharib, M. 1991 Digital particle imaging velocimetry. Exps. Fluids 10, 181-193.

WU, J.Z. 1993 Vorticity dynamics on a fluid-fluid interface. Presented at SIAM Annual Meeting, Philadelphia, PA, July 12-16.

YeH, H. H. 1991 Vorticity generation mechanisms in bores. Proc. R. Soc. Lond. A 432, 215-231. 